\title{
Assessment of Pacing Maneuvers Used to Validate Anterograde Accessory Pathway Potentials
}

\author{
MARK J. NIEBAUER, M.D., Ph.D., EMILE DAOUD, M.D., RAJ GOYAL, M.D., \\ MARK HARVEY, M.D., MARK CASTELLANI, M.D., FRANK BOGUN, M.D., \\ K. KWOK CHAN, M.D., K. CHING MAN, D.O., ADAM STRICKBERGER, M.D., \\ and FRED MORADY, M.D.
}

From the Department of Internal Medicine, Division of Cardiology, The University of Michigan Medical Center, Ann Arbor, Michigan

\begin{abstract}
Validation of Accessory Pathway Potentials. Four pacing maneuvers have been proposed to validate an anterograde accessory pathway potential (APP): (1) atrial pacing to induce complete block between the atrial electrogram and the APP; (2) ventricular pacing to advance the APP without altering the timing of the atrial electrogram; (3) atrial pacing to induce complete block between the APP and the ventricular electrogram; and (4) ventricular pacing to advance the ventricular electrogram without altering the timing of the APP. The purpose of this study was to assess these validation techniques by applying them to electrograms that simulated APPs but which were known to be atrial in origin. In 32 patients undergoing an electrophysiology procedure, a split atrial electrogram containing two components separated by at least $30 \mathrm{msec}$ (mean $54 \pm 15 \mathrm{msec}$ ) was recorded. Using an atrial extrastimulus technique, complete block between the two components of the atrial electrogram (criterion 1) could never be induced, but complete block between the second component of the atrial electrogram and the ventricular electrogram (criterion 3) consistently was induced. Using a ventricular extrastimulus technique, the second component of the atrial electrogram consistently could be advanced by 10 to $40 \mathrm{msec}$ without altering the timing of the first component (criterion 2). In addition, with ventricular pacing, the ventricular electrogram consistently was advanced without altering the timing of the two components of the atrial electrogram (criterion 4). In conclusion, among the four pacing maneuvers used to validate an anterograde APP, the only one that may be specific for an APP is the ability to induce complete block between the atrial electrogram and the APP. (J Cardiovasc Electrophysiol, Vol. 6, pp. 350-356, May 1995)
\end{abstract}

accessory pathway potential, atrial electrogram, Wolff-Parkinson-White syndrome

\section{Introduction}

Accessory pathway potentials can be recorded in the electrophysiology laboratory and have been used to elucidate the anatomy of accessory pathways and to identify target sites for catheter ablation of accessory pathways. ${ }^{1-12}$ However, a local atrial or ventricular electrogram may contain multiple components, one or more of which could be mistaken for an accessory pathway potential.

Address for correspondence: Fred Morady, M.D., University of Michigan Medical Center, 1500 East Medical Center Drive, B1F245, Ann Arbor, MI 48109-0022. Fax: 313-936-7641.

Manuscript received 19 December 1994; Accepted for publication 4 April 1995.
Four pacing maneuvers have been proposed for validating an accessory pathway potential by demonstrating that it is not part of the atrial or ventricular electrogram (Fig. 1) ${ }^{13}$ : (1) atrial pacing to induce complete block between the atrial electrogram and the accessory pathway potential; (2) ventricular pacing to advance the accessory pathway potential without altering the timing of the atrial electrogram; (3) atrial pacing to induce complete block between the accessory pathway potential and the ventricular electrogram; and (4) ventricular pacing to advance the ventricular electrogram without altering the timing of the accessory pathway potential. The specificity of these pacing maneuvers has never been evaluated. 


\section{A. CRITERION 1}

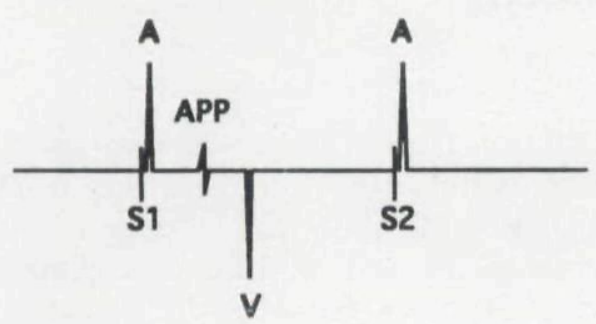

C.

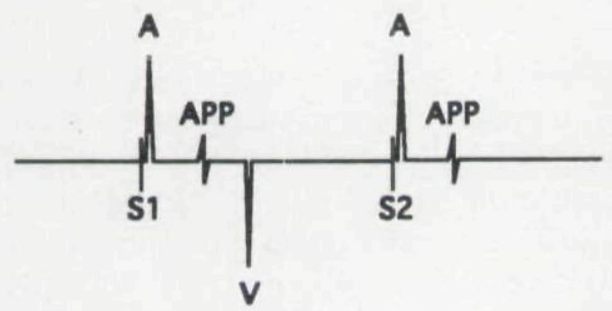

B. CRITERION 2

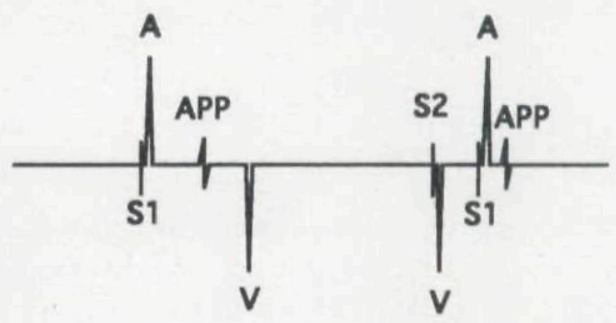

D. CRITERION 4

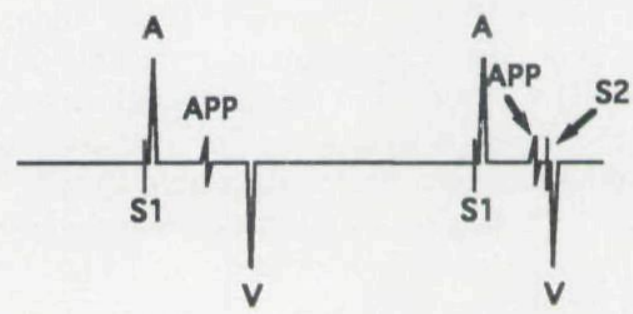

Figure 1. A schematic illustration of the four pacing maneuvers used to validate an anterograde accessory pathway potential. Panel A: Criterion 1. A paced (SI) atrial electrogram (A) is followed closely by a presumed accessory pathway potential (APP). An atrial extrastimulus (S2) induces block between the atrial electrogram (A) and the APP. Panel B: Criterion 2. A ventricular extrastimulus (S2) advances the APP without affecting the timing of the atrial electrogram. Panel C: Criterion 3. An atrial extrastimulus (S2) results in complete block between the APP and the ventricular electrogram (V). Panel D: Criterion 4. A ventricular extrastimulus (S2) advances the ventricular electrogram (V) without affecting the timing of the atrial electrogram or the APP. (Adapted from reference 13.)

Therefore, the purpose of this study was to assess the specificity of these validation techniques by applying them to split electrograms known to be atrial in origin.

\section{Methods}

\section{Patient Characteristics}

The subjects of this study were 32 patients who underwent an electrophysiology procedure at the University of Michigan Medical Center. The selection criteria consisted of the presence of a split atrial electrogram in the right atrium and, in the subjects in whom ventricular pacing was performed, a ventriculoatrial block cycle length shorter than $500 \mathrm{msec}$. The 32 subjects in the study were selected from a group of 50 patients who were screened. There were 22 women and 10 men (mean age $43 \pm 17$ years). None of the patients had structural heart disease. The clinical indication for the electrophysiology procedure was radiofrequency ablation of atrioventricular nodal reentrant tachycardia in 20 patients, accessory pathway ablation in 8 patients, and a diagnostic electrophysiology test in 4 patients.

\section{Electrophysiologic Testing}

The electrophysiology procedures were performed in the fasting state after informed consent was obtained and after all antiarrhythmic medications had been withheld for at least five halflives. Three quadripolar electrode catheters were inserted into a femoral vein and positioned in the right atrium, across the tricuspid valve to record the His-bundle electrogram, and in the right ventricular apex. Leads $\mathrm{V}_{1}, \mathrm{I}$, II, and III and the intracardiac electrograms were displayed on an oscilloscope and recorded on paper at $100 \mathrm{~mm} / \mathrm{sec}$ with a Mingograph-7 recorder (Siemens-Elema, Solna, 
Sweden). Programmed stimulation was performed with a programmable stimulator (Bloom Associates, Reading PA, USA).

\section{Study Protocol}

The study protocol was performed upon completion of the clinically indicated portion of the electrophysiology procedure. In the patients who underwent an ablation procedure, the study protocol was performed after successful ablation, during the 30 -minute waiting period to confirm a successful outcome. A quadripolar electrode catheter with a 2-mm interelectrode spacing for the distal pair of electrodes was used to search for a split atrial electrogram that had two components separated by an isoelectric segment in the high or midlateral right atrium or in the right atrial appendage. The electrograms were filtered at a bandpass of 50 to $500 \mathrm{~Hz}$ and recorded at a gain of 40 to 80 $\mathrm{mm} / \mathrm{mV}$. Sixteen patients underwent ventricular pacing maneuvers and another 16 patients underwent atrial pacing maneuvers.

In the patients who underwent atrial pacing maneuvers, an electrode catheter was positioned against the high lateral right atrium, superior to the catheter being used to record the split atrial electrogram. Using basic drive trains of eight beats at a cycle length of 400 to $600 \mathrm{msec}$, diastole was scanned with a single atrial extrastimulus in decrements of $10 \mathrm{msec}$ to the point of atrial refractoriness, in order to assess criteria 1 and 3 (Figs. 1A and $1 \mathrm{C}$ ).

In the patients who underwent ventricular pacing maneuvers, an electrode catheter was positioned against the high lateral right atrium, superior to the catheter being used to record the split atrial electrogram. Continuous atrial pacing was performed at a cycle length $50 \mathrm{msec}$ shorter than the sinus cycle length in order to eliminate variability in the atrial cycle length. Diastole was then scanned in 10-msec decrements with a ventricular extrastimulus introduced every fifth cycle. This maneuver allowed assessment of criteria 2 and 4 (Figs. 1B and 1D).

\section{Data Analysis}

Values are expressed as mean $\pm \mathrm{SD}$. Comparisons were performed using the Student's paired $t$-test. A P value $<0.05$ was considered statistically significant.

\section{Results}

\section{Criterion 1}

Criterion 1 could not be demonstrated in any patient. Atrial extrastimulus testing resulted in an increase in the interval between the two components of the split atrial electrogram from a mean of $50 \pm 14 \mathrm{msec}$ to $77 \pm 21 \mathrm{msec}(\mathrm{P}<$ $0.005)$, but complete block between the two components of the electrogram was never induced (Fig. 2).

\section{Criterion 2}

Criterion 2 was demonstrated in every patient who underwent ventricular extrastimulus testing. With appropriately timed ventricular extrastimuli that conducted retrograde through the atrioventricular node, the second component of the split atrial electrogram was advanced by 10 to $40 \mathrm{msec}$ without altering the timing of the first component (Fig. 3). The mean interval between the two components of the split atrial electrogram was shortened from $55 \pm 15$ msec to a minimum of $34 \pm 16 \mathrm{msec}(\mathrm{P}<$ $0.005)$.

\section{Criterion 3}

Criterion 3 was demonstrated in each of the 16 patients in whom atrial extrastimulus testing was performed. At the effective refractory period of the atrioventricular node, there was block between the second component of the split atrial electrogram and the ventricular electrogram (Fig. 4).

\section{Criterion 4}

Criterion 4 also was demonstrated in every patient in whom ventricular extrastimulus testing was performed. A ventricular extrastimulus late in diastole consistently resulted in advancement of the ventricular depolarization recorded in the right atrium by 25 to $50 \mathrm{msec}$ without altering the timing of the two components of the split atrial electrogram (Fig. 5). 


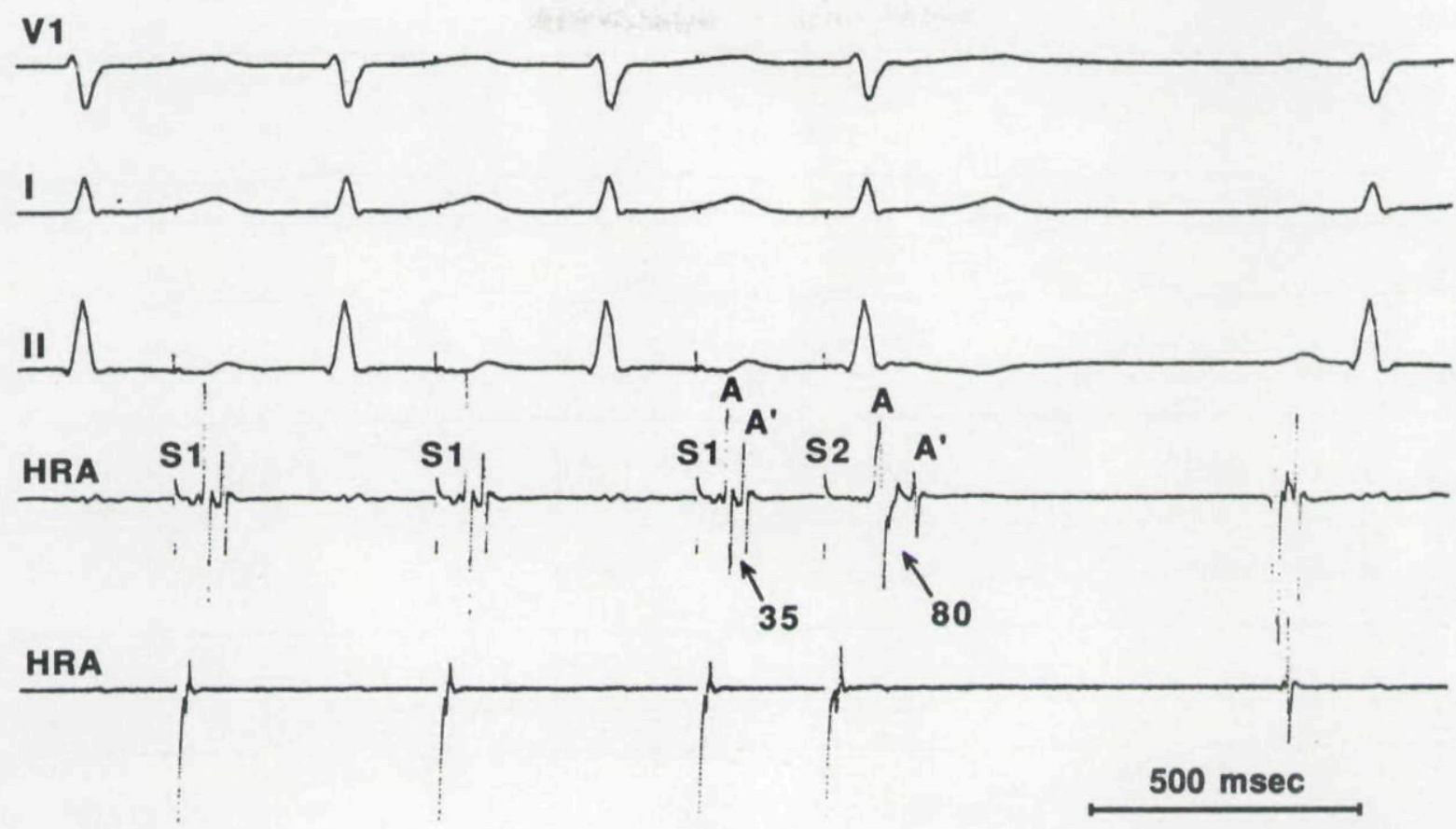

Figure 2. Evaluation of criterion 1. Shown are leads V1, I, and II, a split atrial electrogram recorded in the high right atrium (HRA), and an electrogram recorded from a pacing catheter in the HRA. The split atrial electrogram consists of two components $\left(A\right.$ and $\left.A^{\prime}\right)$; note that $A^{\prime}$ simulates an accessory pathway potential. Scanning diastole with an atrial extrastimulus (S2) in 10-msec decrements to the point of atrial refractoriness resulted in an increase in the A-A' interval from a baseline value of 35 msec to a maximum of 80 msec. However, complete block between A and $A^{\prime}$ could not be induced in this or any other patient in whom this maneuver was evaluated.

\section{Discussion}

\section{Validation of Accessory Pathway Potentials}

Because an accessory pathway potential occurs before the onset of the QRS complex and ventricular electrograms recorded near the tricuspid or mitral annulus usually do not, it is unlikely that the first component of a split ventricular electrogram would occur early enough to simulate an accessory pathway potential. On the other hand, the second component of a split atrial electrogram recorded near the tricuspid or mitral annulus could easily simulate an accessory pathway potential. Therefore, validation criteria for accessory pathway potentials are necessary. The pacing techniques that have been used to validate accessory pathway potentials have been intended to demonstrate that the depolarization in question arises from a different anatomic structure than do the local atrial and ventricular depolarizations. ${ }^{13}$

The results of the present study demonstrate that among the four pacing maneuvers that have been used to validate anterograde accessory pathway potentials, the only one that may be specific for an accessory pathway potential is the ability to induce complete block between the atrial electrogram and the accessory pathway potential. The other three criteria can be consistently demonstrated with a split atrial electrogram and, therefore, should not be considered specific for an accessory pathway potential. In particular, the ability to advance a depolarization without altering the timing of a closely preceding depolarization (criterion 2) does not indicate that the two depolarizations arise from different anatomic structures. Although the two depolarizations undoubtedly arise from different myocardial cells or groups of cells, the results of this study demonstrate that two depolarizations arising from the same anatomic structure, i.e., the right atrium, can be dissociated from each other.

\section{Split Atrial Potentials}

The split atrial potentials recorded in the patients in this study provided an ideal model for testing the specificity of validation criteria for accessory pathway potentials because they closely simulated the appearance of accessory pathway 

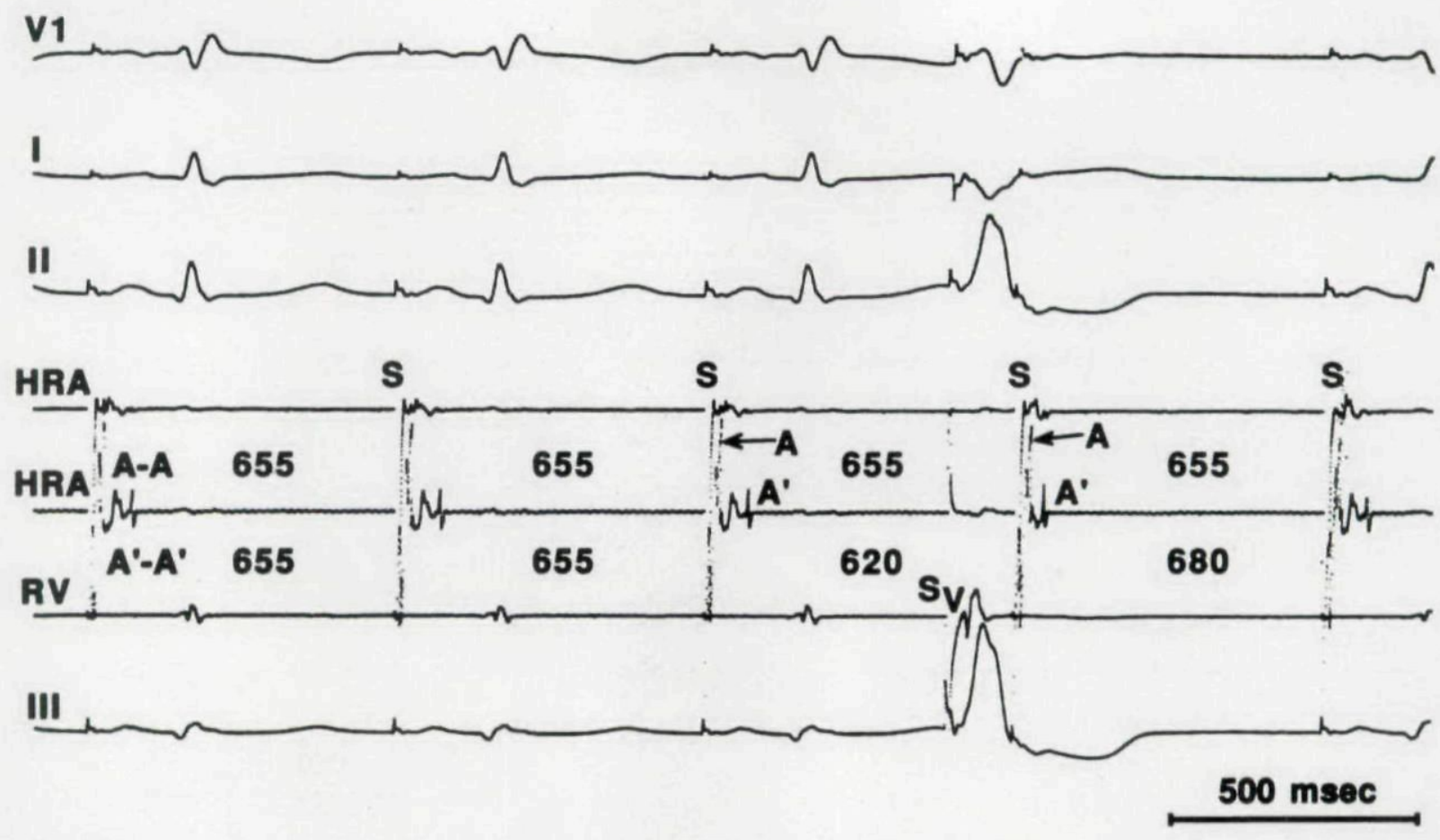

Figure 3. Evaluation of criterion 2. Shown are leads VI, I, and II, a high right atrial electrogram (HRA), a split atrial electrogram recorded in the high right atrium (HRA), a right ventricular electrogram $(R V)$, and lead III. Note that the second component of the split atrial electrogram $\left(A^{\prime}\right)$ simulates an accessory pathway potential. The RA was paced at a cycle length of $655 \mathrm{msec}$, and a ventricular extrastimulus (SV) was induced in diastole. The coupling interval of the ventricular extrastimulus was shortened in 10-msec steps until the second component of the split atrial electrogram ( $\left.A^{\prime}\right)$, but not the first component (A), was advanced. The intervals between consecutive first and second components $\left(A-A\right.$ and $\left.A^{\prime}-A^{\prime}\right)$ are shown in milliseconds. In this patient, $A^{\prime}$ was advanced by $35 \mathrm{msec}$ without any change in the timing of $A$.

potentials and yet were recorded at sites several centimeters from the tricuspid annulus, assuring that they were, in fact, atrial in origin. Although the mechanism of these electrograms is not clearly established, many of the split atrial electrograms used in this study were recorded in the high lateral right atrium, suggesting a relationship to the crista terminalis; for example, it is possible that the crista terminalis resulted in discontinuity in local atrial activation. However, several of the split atrial electrograms were recorded at sites away from the crista terminalis, indicating that other mechanisms also were operative. McGuire et al. ${ }^{14}$ demonstrated that double atrial potentials recorded in the posterior septum result from asynchronous activation of different layers of atrial cells. It is possible that the complex geometric arrangement of atrial cells in other areas of the atrium also could account for a split electrogram, including areas near the mitral and tricuspid valve rings where accessory pathway potentials are recorded. Indeed, McGuire et al. ${ }^{14}$ recorded split atrial potentials around the tricuspid annulus and documented transitional nodal-type cells at those locations.

\section{Conclusions}

The results of this study demonstrate that the only pacing maneuver which may be helpful in validating an anterograde accessory pathway potential is atrial pacing that results in conduction block between the atrial electrogram and the presumed accessory pathway potential. However, although this criterion was never met with the split atrial electrograms recorded in this study, the possibility that complete block between the two components of a split atrial electrogram occasionally might be inducible cannot be ruled out. In regards to the three other pacing maneuvers that have been used for validation of anterograde accessory pathway potentials, these are nonspecific and may not be helpful in distinguishing an accessory pathway potential from a component of a split atrial electrogram. It may be that the most reliable criterion for validation of an anterograde accessory 


\section{V1}

I

II
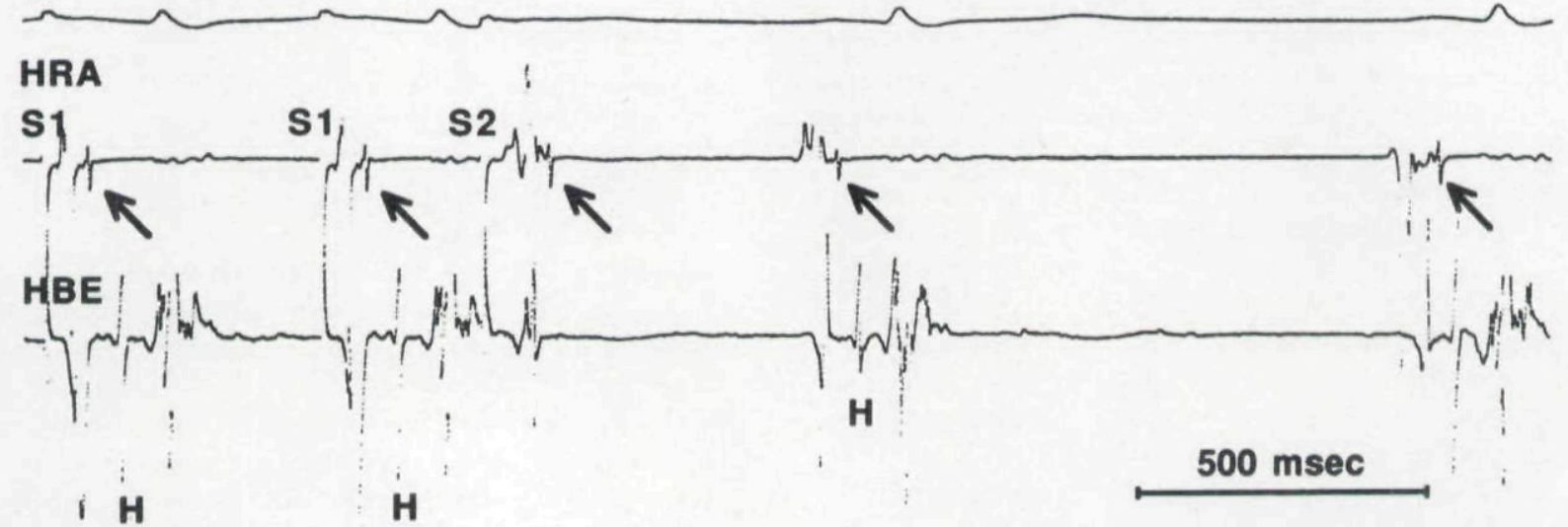

Figure 4. Evaluation of criterion 3. Shown are leads VI, I, and II, a split atrial electrogram recorded in the high right atrium (HRA), and the His-bundle electrogram (HBE). The second component of the split atrial electrogram is indicated by an arrow and simulates an accessory pathway potential. Following an 8-beat basic drive train (S1) at a cycle length of 500 msec, an atrial extrastimulus (S2) results in an atrial depolarization that does not conduct to the ventricle. Therefore, block was induced between the simulated accessory pathway potential and the ventricular electrogram. $H=H i$-bundle potential.

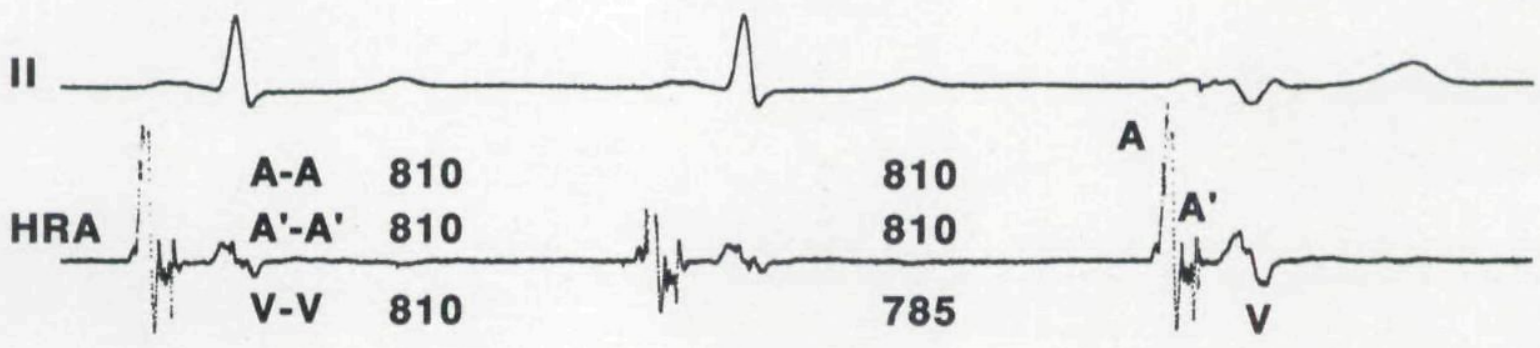

RV

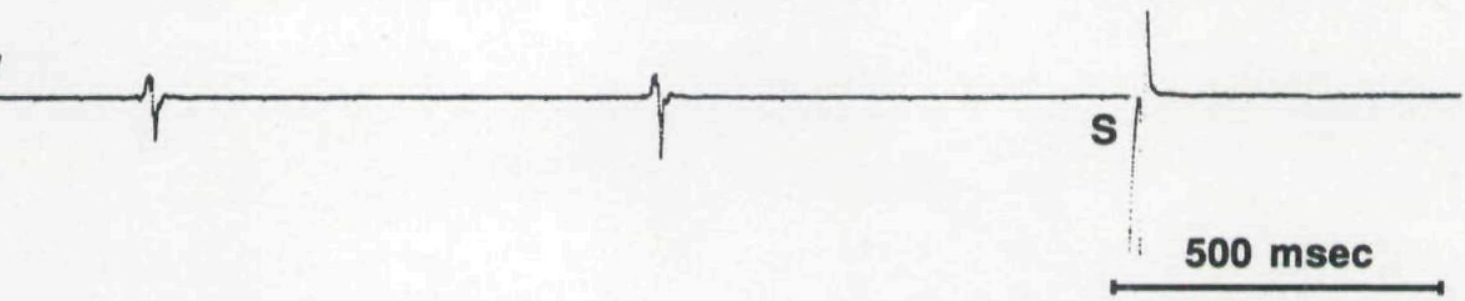

Figure 5. Evaluation of criterion 4. Shown are lead II, a split atrial electrogram recorded in the high right atrium (HRA), and a right ventricular electrogram $(R V)$. A ventricular extrastimulus $(S)$ was induced in late diastole. The ventricular electrogram recorded in the HRA was advanced by $25 \mathrm{msec}$ without alteration in the timing of the two components of the atrial electrogam $\left(A\right.$ and $\left.A^{\prime}\right)$. The intervals between consecutive corresponding electrograms $\left(A-A, A^{\prime}-A^{\prime}\right.$, and $V$-V) are shown in milliseconds. 
pathway potential is its presence during preexcitation and its absence when there is not preexcitation. $^{1}$

\section{References}

1. Prystowsky EN, Browne KF, Zipes DP: Intracardiac recording by catheter electrode of accessory pathway depolarization. J Am Coll Cardiol 1983;1:468-470.

2. Schluter M, Geiger M, Siebels J, et al: Catheter ablation using radiofrequency current to cure symptomatic patients with tachyarrhythmias related to an accessory atrioventricular pathway. Circulation 1991:84:1644-1661.

3. Jackman WM, Wang X, Friday KJ, et al: Catheter ablation of accessory atrioventricular pathways (WolffParkinson-White Syndrome) by radiofrequency current. N Engl J Med 1991;324:1605-1611.

4. Chen X, Borggrefe M, Shenesa M, et al: Characteristics of local electrogram predicting successful transcatheter radiofrequency ablation of left-sided accessory pathways. J Am Coll Cardiol 1992;20:656-665.

5. Calkins H, Kim Y-N, Schmaltz S, et al: Electrogram criteria for identification of appropriate target sites for radiofrequency catheter ablation of accessory atrioventricular connections. Circulation 1992:85:565-573.

6. Lesh MD, Van Hare GF, Schamp DJ, et al: Curative percutaneous catheter ablation using radiofrequency energy for accessory pathways in all locations: Results in 100 consecutive patients. J Am Coll Cardiol 1992;19:1303-1309.

7. Winters SL, Gomes JA: Intracardiac electrode catheter recordings of atrio-ventricular bypass tracts in WolffParkinson-White syndrome: Techniques, electrophysiologic characteristics and demonstration of concealed and decremental propagation. J Am Coll Cardiol 1986; 7:1392-1403.

8. Silka MJ, Kron J, Halperin BD, et al: Analysis of local electrogram characteristics correlated with successful radiofrequency catheter ablation of accessory atrioventricular pathways. PACE 1992;15:1000-1007.

9. Hassaguierre M, Fischer B, Waren J-F, et al: Electrogram patterns predictive of successful radiofrequency catheter ablation of accessory pathways. PACE 1992;15:2138-2145.

10. Calkins H, Langberg J, Sousa J, et al: Radiofrequency catheter ablation of accessory atrioventricular connections in 250 patients. Abbreviated therapeutic approach to Wolff-Parkinson-White syndrome. Circulation 1992;85:1337-1346.

11. Swartz JF, Tracy CM, Fletcher RD: Radiofrequency endocardial catheter ablation of accessory atrioventricular pathway atrial insertion sites. Circulation 1993;87:487-499.

12. Deshpande SS, Bremner S, Sra JS, et al: Ablation of left free-wall accessory pathways using radiofrequency energy at the atrial insertion site: Transseptal versus transaortic approach. J Cardiovasc Electrophysiol 1994;5:219-231.

13. Jackman WM, Friday KJ, Yeung-Lai-Wah JA, et al: New catheter technique for recording left free-wall accessory atrioventricular pathway activation: identification of pathway fiber orientation. Circulation 1988:78:598-610.

14. McGuire MA, deBakker MT, Vermeulen JT, et al: Origin and significance of double potentials near the atrioventricular node. Correlation of extracellular potentials, intracellular potentials, and histology. Circulation 1994;89:2351-2360. 
This document is a scanned copy of a printed document. No warranty is given about the accuracy of the copy. Users should refer to the original published version of the material. 